\title{
Feature extraction of jujube fruit wrinkle based on the watershed segmentation
}

\author{
Zhang J unxiong*, Ma Qingqing, Li Wei, Xiao Tingting \\ (College of Engineering, China Agricultural University, Beijing 100083, China)
}

\begin{abstract}
The degree of surface wrinkles on a dried jujube fruit (Ziziphus jujuba Mill.) is an important quality grading criterion. The aim of this research was to propose an image processing method based on the watershed segmentation to extract the wrinkle features of jujube fruits. Original images of jujube fruit taken under cyan light were transformed into grayscale images. The noise in these images was then removed by morphological reconstruction. The H-minima extended transformation was used to label the foreground of jujube fruit images after reconstruction, and the labeled foreground regions were segmented by a distance transform-based watershed algorithm. Then, the grayscale images were filtered with a local range filter. The segmentation function was obtained using the minima imposition method. Finally, a watershed segmentation was used to extract the wrinkle features of jujube fruits. Experiments on 304 images of jujube fruit showed that the accuracy of wrinkle-based grading obtained by the algorithm was $92.11 \%$, which proved that this method could be used to classify jujube wrinkles.
\end{abstract}

Keywords: feature extraction, watershed segmentation, image processing, jujube fruit, wrinkle, quality grading DOI: $10.25165 /$ j.ijabe.20171004.2638

Citation: Zhang J X, Ma Q Q, Li W, Xiao T T. Feature extraction of jujube fruit wrinkle based on the watershed segmentation. Int J Agric \& Biol Eng, 2017; 10(4): 165-172.

\section{Introduction}

The surface of the jujube fruit becomes increasingly wrinkled when it is dried, and the use of jujubes differs according to the degree of wrinkles. Dried red jujubes with more wrinkles are usually used in medicine, while the jujubes with smooth surfaces are used in foods such as preserves.

In recent years, jujube grading systems based on digital image processing have been studied. Jujubes are native to China, but in other countries (except Korea), jujube is only limited to noncommercial cultivation or

\section{Received date: 2016-06-12 Accepted date: 2016-10-06}

Biographies: Ma Qingqing, MS, research interests: agricultural robotics and machine vision, Email: 18811782276@163.com; Li Wei, PhD, Professor, research interests: agricultural robotics, Email: liww@cau.edu.cn; Xiao Tingting, MS, research interests: agricultural robotics and machine vision, Email: xiaotingting0214@163.com.

*Corresponding author: Zhang Junxiong, PhD, Associate Professor, research interests: agricultural robotics. College of Engineering, China Agricultural University, Beijing 100083, China. Tel: +86-13466369619, Email: cau2007@cau.edu.cn. research $^{[1-3]}$. There are hence few studies on jujube grading and detection technologies outside of China. For date palm fruits, Dah-Jye et al..$^{[4,5]}$ used size and maturity as classification indexes for grading. In their studies, the maturity index was determined by the color of the date palm's surface, and methods for color space conversion and color distribution analysis were proposed. A more robust linear system to calibrate color with respect to maturity was built. Ohali ${ }^{[6]}$ used external quality as the characteristic index for date palm fruits. RGB images of dates which were primary prepared were detected by a backpropagation algorithm with a classification accuracy of $80 \%$. Xue et al. ${ }^{[7]}$ used hyperspectral technology to detect a Huping jujube's external "natural defects" and internal "soluble solid contents" simultaneously. Most jujube grading detection systems focus on features such as shape, defects, and internal qualities such as soluble solid content ${ }^{[8-11]}$. Wrinkle texture is one of the most important features of a jujube, but there are fewer studies that address it. Wang $^{[12]}$ extracted the wrinkle features on the surface of a 
jujube using a wavelet transform combined with morphological operations. However, the wrinkle texture was not extracted completely. Only the deeper wrinkles that cannot be used as a basis for grading were detected. A comparison of Roberts, Sobel, Prewitt, and Laplace operators for detecting jujube wrinkle features found that the Laplacian 4-adjacency operator was faster and more accurate, and hence suitable for grading based on wrinkle classification $^{[13]}$. Zhan ${ }^{[14]}$ used six statistical features of a gray level histogram: mean, standard deviation, $R$ (normalized), the third moment, uniformity, and entropy, and combined them with a backpropagation neural network to classify texture on jujubes. He also calculated the threshold ranges for grading. The practical application of this method is relatively narrow because wrinkles on jujube are complex and varied. In short, it is necessary to extract wrinkle features on a jujube as a basis for grading systems. This process is a key to improving jujube grading systems.

Wrinkles on jujube are a kind of natural texture with irregularly distributed features. In contrast to the defects and classification features of other fruits, a wrinkle on the surface of a jujube is a kind of spatial texture and the colors of the wrinkled and smooth regions are not obviously different. In particular, regions with wrinkles consist of sunken "valleys" surrounded on both sides by raised "ridges." In contrast to smooth regions, the appearance of wrinkles' brightness in the image will change when environmental conditions such as the light source are changed.

In image processing, the watershed segmentation, which is a mathematical morphological algorithm based on topology theory, is an effective way to extract the target regions of remote sensing information and multiple objects $^{[15,16]}$. When objects in an image are touching, like the wrinkles of a jujube, they are difficult to separate. The watershed transform is often used to solve this problem. The watershed transform finds "catchment basins" and "watershed ridge lines" in an image by treating it as a surface where the light pixels are taken as high elevations and dark pixels are taken as low elevations. Segmentation using the watershed transform works better if it is possible to identify or mark foreground objects and background locations. A jujube-fruit wrinkle feature extraction method based on a marked watershed segmentation is proposed in this paper. This method is used for segmenting the wrinkles of a single object under cyan lighting.

\section{Materials and methods}

\subsection{Materials}

\subsubsection{Samples and experimental setup}

Jujube fruit of different sizes without obvious defects were used to create the images for the experiments. They were harvested in the city of Hotan in Xinjiang Uygur Autonomous Region in October, 2015. The individual fruit were preliminarily divided by hand into three grades according to the degree of wrinkles. Figure 1 shows that the first grade of jujube fruit were smooth with surfaces that had gentle pits. The wrinkles of the second grade were obvious but moderate, and the wrinkles of the third grade were much more intense and were both dense and deeper than those of the other grades.

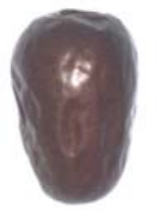

a. Grade 1

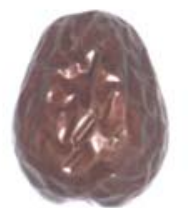

b. Grade 2

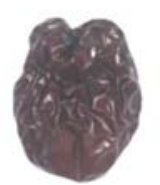

c. Grade 3
Figure 1 Jujube fruit grades according to degree of winkles

The hardware of the image acquisition system was composed of a structural frame, industrial camera, computer, illuminant, light controller, camera lens and imaging surface. The software platform is Matlab R2014a.

Industrial camera MER-200-14GC (China Daheng (Group) Co. Ltd.) is a color CCD camera with resolution of $1628 \times 1236(\mathrm{H} \times \mathrm{V})$ and a frame rate of $14 \mathrm{fps}$. Camera lens OPT-C2514-5M (Dongguan OPT Automation Technology Co., Ltd.) is a C-mount lens with $25 \mathrm{~mm}$ focal length. Light source OPT-RI3C6215 (Dongguan OPT Automation Technology Co., Ltd.) is a RGB trichromatic LED light source.

\subsubsection{Experimental procedure and light environment}

To acquire a test image, a single jujube fruit was placed on the imaging surface, which has a white background. The camera was adjusted to a suitable 
height $(31 \mathrm{~cm}$ high above the imaging surface for a $25 \mathrm{~mm}$ focal length lens). Then, the jujube fruit was moved to the center of the field of view. Figure 2a shows that the wrinkles were not clear under a white light. Hence, other colored lights were considered in this study. By adjusting the light controller, jujube images were acquired under six light colors: red, green, blue, and their corresponding secondary colors, cyan (green + blue), magenta (red + blue), and yellow (red + green). Example images are shown in Figure 2b. All image groups were processed using the algorithm described below: Table 1 shows the numbers of wrinkles obtained for each group. Local region $A$ of the jujube fruit image in Figure 2a is magnified and wrinkle texture recognitions under the six light colors are shown in Figure 2c. It is clear that the wrinkle texture of the jujube fruit under cyan light is the clearest and the image contains many more details than the others. Therefore, cyan light was used to acquire the jujube fruit images in the following experiments.

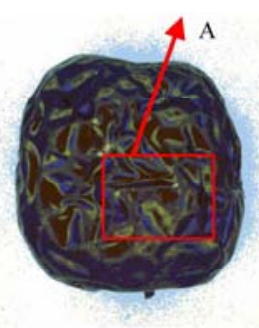

a. Real image

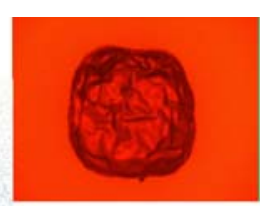

b1. Red light

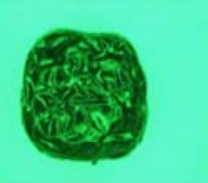

b2. Green light

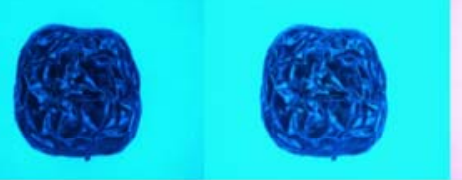

b3. Blue light b4. Cyan light

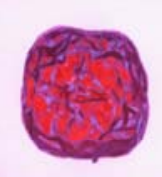

b5. Magenta light

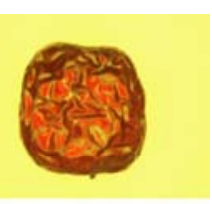

b6. Yellow light

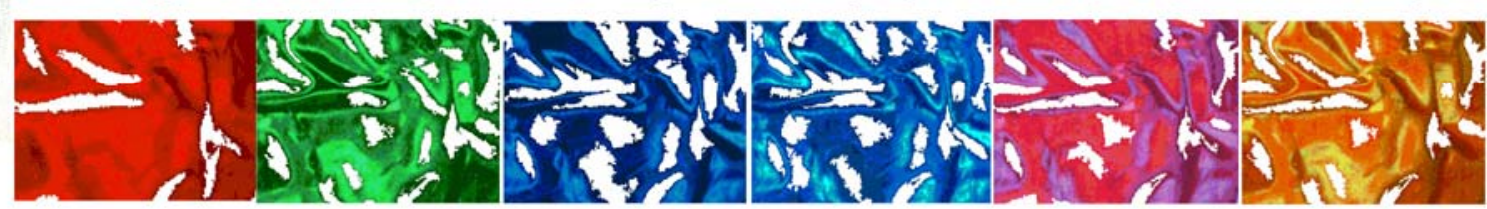

cl. Red light

c2. Green light

c3. Blue light

c4. Cyan light

c5. Magenta light

c6. Yellow light

Figure 2 Comparison of jujube fruit images under six color lights

Table 1 Number of wrinkles imaged under six light colors

\begin{tabular}{ccccccc}
\hline \multirow{2}{*}{ Label } & \multicolumn{6}{c}{ Light } \\
\cline { 2 - 7 } & Red & Green & Blue & Cyan & Magenta & Yellow \\
\hline 1 & 27 & 70 & 63 & 75 & 58 & 67 \\
2 & 24 & 72 & 63 & 71 & 50 & 64 \\
3 & 13 & 53 & 46 & 60 & 41 & 48 \\
4 & 17 & 57 & 55 & 62 & 47 & 50 \\
5 & 48 & 120 & 107 & 121 & 87 & 109 \\
6 & 44 & 103 & 94 & 109 & 76 & 99 \\
\hline
\end{tabular}

Another important factor that influences the details of the wrinkles in the jujube images is the light intensity. The camera's exposure time was set to $2250 \mu \mathrm{s}$ and the aperture coefficient was adjusted to F1.4. The light controller was then adjusted to control the brightness of the light and an illuminometer was placed on the imaging surface directly below the LED light to measure the light intensity. Five images were collected by adjusting the knob on the light controller to 2, 2.25, 2.5, 2.75 and 3, and the effect was evaluated using the grayscale mean variance and grayscale information entropy ${ }^{[17]}$. The curve in Figure 3 was obtained by fitting the results, which shows that the best light intensity in this experiment is $25000-45000 \mathrm{~lx}$. Hence, the light intensity was set to $35800 \mathrm{~lx}$ in the following experiments.

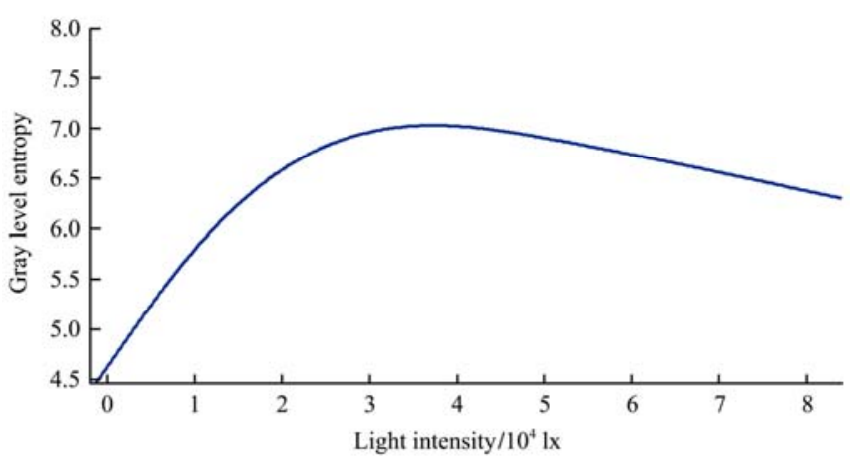

a. Grayscale information entropy

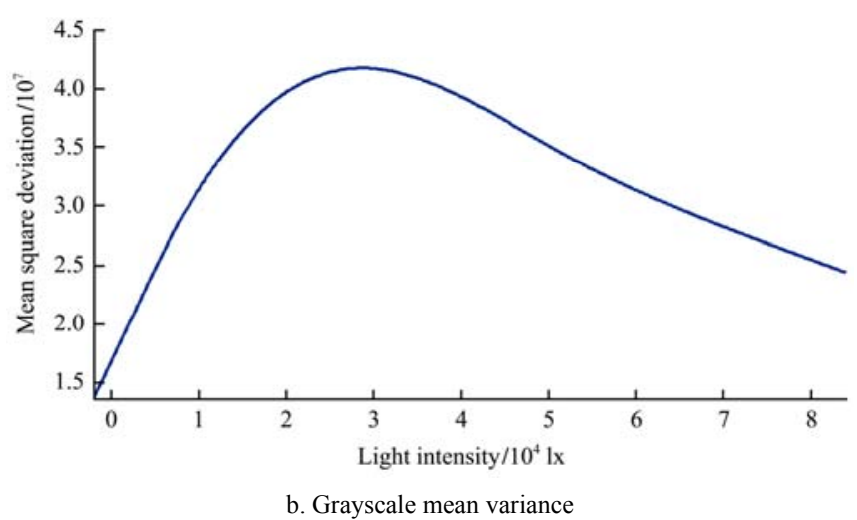

Figure 3 Effects of light intensity on the obtained images

\subsection{Methods}

\subsubsection{Morphological de-noising}

First, the original image (Figure 4a) was transformed into a grayscale image (Figure $4 b$ ). This figure shows 
that many white noise pixels exist in the dark wrinkle regions and many black noise pixels exist in the light smooth regions. The presence of noise causes the watershed algorithm to seriously over-segment the image. Hence, it is necessary to remove these noisy pixels from the grayscale image. In contrast to filters such as average or Laplace filters, a morphological filter can enhance the edges of images and remove noise simultaneously. It is helpful for target extraction if the edges within images are enhanced.

Bright noise areas that are smaller than a morphological structuring element can be removed by an opening operation and small dark noise areas that are smaller than a morphological structuring element can similarly be removed by a closing operation. The targets in the image that should be preserved after erosion and dilation can be accurately preserved by a morphological opening and closing reconstruction. Therefore, the grayscale images are morphologically reconstructed before they are labeled. The structuring element is a "disk" of radius 5 pixels. The specific steps of this procedure are given by the following Equations:

$$
\begin{gathered}
f^{\prime}(x, y)=R_{f(x, y)}(f(x, y) \ominus B) \\
f^{\prime \prime}(x, y)=f^{\prime}(x, y)^{C} \# \# \# \# \# \# \# \# \# \# \text { (2)\# } \\
g(x, y)=f^{\prime \prime}(x, y) \oplus B \\
g^{\prime}(x, y)=\left(\operatorname{Rg}(x, y)\left(g(x, y) \ominus f^{\prime \prime}(x, y)\right)\right)^{C}
\end{gathered}
$$

In these equations, $f(x, y)$ is the grayscale image; $g^{\prime}(x, y)$ is the reconstructed image; $\oplus$ indicates the dilation operation; $\ominus$ denotes the erosion operation; $C$ is the complement of the image; and $R$ is the reconstruction operation.

The final image de-noised by opening and closing reconstruction is shown in Figure 4c.

\subsubsection{Targets and background labeling}

Related and unrelated features of an image can be labeled by the extremum of a region. Using a contrast criterion, the H-minima transform ${ }^{[18]}$ inhibits all minima whose depths are equal to or larger than a threshold $h$. The erosion and reconstruction of $f(x, y)$ is computed by $(f(x, y)+h)$. The value of the extended minimum EMIN is the minimum of the region corresponding to the $H$-minima transform.

$$
\operatorname{EMIN}_{h}(f(x, y))=\operatorname{RMIN}\left[R_{f(x, y)}^{\delta}(f(x, y)+h]\right.
$$

where, EMIN denotes the extended minima; RMIN denotes the $h$-minima transform; $\delta$ is dilation; $R$ is the reconstruction operation; and $h$ is the threshold.

The lowest grayscale point in every wrinkle region on the surface of the jujube fruit is marked as $M$, and the height threshold is set to $h$. The numbers of the grayscale values in $[M, M+h]$ through the extended minimum transformation are labeled as one region. In other words, "concave" regions, which describe the wrinkles on the surface of the jujube fruit, are labeled well. The grayscale regions shown in Figure $4 \mathrm{~d}$ are target regions that have been labeled. The experiments show that, for jujube fruit wrinkle extraction, it is better to set threshold $h$ to 5 .

Figure $4 \mathrm{e}$ shows an image in which the background has been marked using the target marking that these target markers were limited to the allowable local minimum by distance transforming watershed segmentation. Moreover, the watershed crest lines that go through the highest points between adjacent target markers can be determined.

\subsubsection{Wrinkle feature extraction}

(1) Local range filtering

The purpose of extracting texture features is to highlight the details of the image using image processing. The grayscale levels of the pixels in the wrinkles have large changes in their spatial distribution properties. This kind of spatial structure can inherently be represented by correlation with neighbor pixels. Therefore, the local ranges ${ }^{[19]}$ of the counted images were used to filter the images and the difference between the maximum and minimum value in neighborhood $N$ of a pixel is taken as the value of the central element. This process is given by:

$$
\begin{gathered}
M A X=\max \left\{f\left(x-x^{\prime}, y-y^{\prime}\right) \mid\left(x^{\prime}, y^{\prime}\right) \in N\right\} \\
M I N=\min \left\{f\left(x+x^{\prime}, y+y^{\prime}\right) \mid\left(x^{\prime}, y^{\prime}\right) \in N\right\} \\
f(x, y)=M A X-M I N
\end{gathered}
$$

where, MAX is the maximum in the neighborhood $N$; MIN is the minimum in neighborhood $N ; f(x, y)$ is the value of the central element in the neighborhood $N$.

The image's low-frequency information can be preserved well and the edge contrasts in a region can be 
enhanced if local range filtering is used with a window size of $5 \times 5$. Preliminary wrinkle features with a certain grayscale gradient can be found by filtering. The gradient magnitude image is shown in Figure $4 \mathrm{~g}$.

(2) Minima imposition

Minima imposition ${ }^{[20]}$ is one of the most appropriate methods for avoiding over segmentation produced by the watershed algorithm. For this method, it is necessary to find a pair of suitable markers and the gradient magnitude image. Markers are roughly located on the connected components of the target and background. The gradient magnitude has a high value along the edge of the detected target. The marker is a two times pretreatment that it contains target markers and background markers of gradient magnitude images. The process was given for all pixels by:

$$
\begin{gathered}
f_{m}(x)=\left\{\begin{array}{l}
0, \text { if } x \text { belongs to a marker } \\
t_{\max }, \text { else }
\end{array}\right. \\
f_{2}=R_{\left(f_{1} \wedge f_{m}\right)}^{\varepsilon}\left(f_{m}\right)
\end{gathered}
$$

where, $f_{m}(x)$ is label image; $t_{\max }$ is the grayscale maximum; $f_{1}$ is the gradient magnitude image; $f_{2}$ is the segmentation function; $\Lambda$ is a point-wise minima operation (infimum operation), and $\varepsilon$ is erosion.

The label image is defined as:

$$
f_{m}(p)=O(p) \cup B(p)
$$

where, $O(p)$ denotes the target markers; $B(p)$ denotes the background markers; and $U$ is union.

The labeled image $f_{m}(p)$ is shown in Figure $4 \mathrm{f}$. The labeled image, which is regarded as a local minimum set, is superimposed over the gradient magnitude image in Figure 4g. Then, the gradient magnitude is converted to the segmentation image as shown in Figure $4 \mathrm{~h}$.

(3) Watershed segmentation

The original watershed transformation was applied to the segmented image. The final segmentation image for extracting the wrinkle features is shown in Figure 4i. Figure 5 shows the images of the wrinkle regions separated from the jujube fruit, where most of the wrinkles in the real image correspond to the white wrinkles in Figure 5a. The extracted regions of the wrinkles are superimposed on the original image to obtain Figure $5 \mathrm{~b}$, in which the red pixels represent the wrinkle regions.

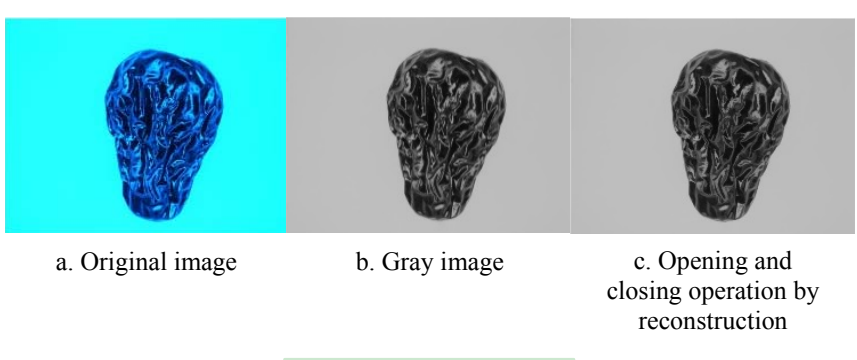

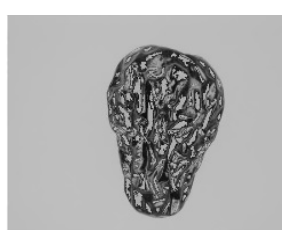

d. Target markers

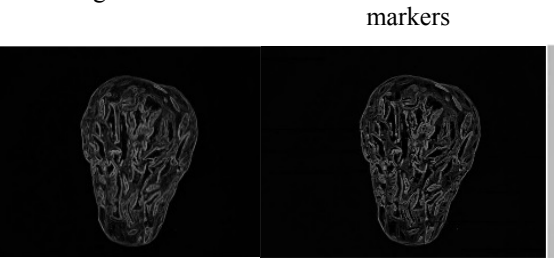

g. Gradient magnitude image

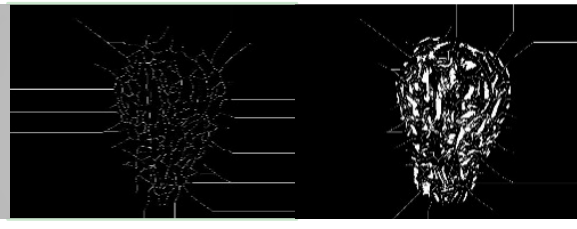

f. Label image

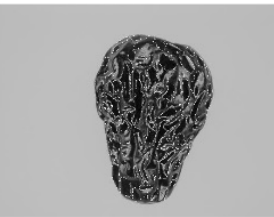

i. Segmented image
Figure 4 Extraction algorithm for wrinkle features

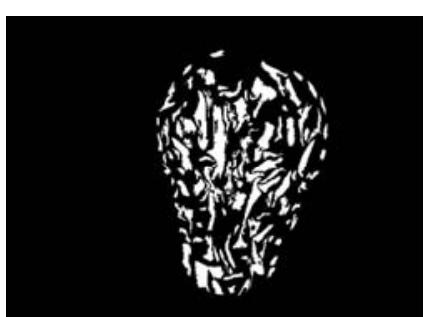

a. Wrinkle separation

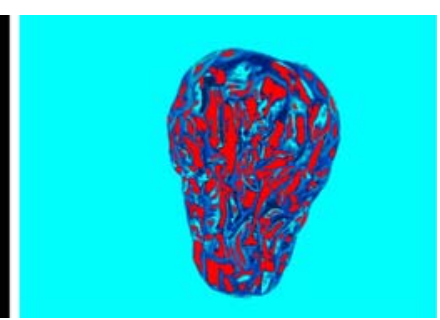

b. Wrinkle superimposition
Figure 5 Wrinkle images

\section{Results and discussion}

\subsection{Determination of the classification threshold}

Firstly, equal numbers of the three grades of jujube fruit were uniformly mixed together. Next, 60 samples were selected randomly and their images were taken. The images were then processed with the algorithm proposed in this study and the segmented regions were counted. At present, a classification standard for jujube wrinkle texture could not be found. However, before classification, standard samples were prepared by referencing specifications and test methods for the shape and color of jujube fruit appearance ${ }^{[21]}$. In this study, the 60 jujube fruit were classified into three grades according to the number of wrinkles using a $K$-means clustering algorithm. Two fruit samples, named $\mathrm{A}$ and $\mathrm{B}$, from the classification boundaries were chosen to be standard samples (Figure 6) and the number of the wrinkles on A and B (76 and 118, respectively) 
determined the classification thresholds for the clustering results. In addition, asterisks in Figure 6 indicate the positions of the clusters' centers of mass.

\subsection{Classification experiments}

The chosen jujube fruit samples were put on a clean surface according to the national jujube classification standard GB/T 5835-2009. Then, the degree of wrinkles on the jujube fruit were determined by comparison with standard jujube fruit samples $A$ and $B$. The classification criterion was as follows: A fruit that was smoother than jujube fruit $\mathrm{A}$ was classified as the first-level grade. If it was more wrinkly than jujube fruit $\mathrm{B}$, it was classified as third-level grade. In contrast, a fruit whose degree of wrinkles fell between the first-level and third-level grades was classified as second-level grade. Next, 304 jujube fruit were graded automatically by this algorithm. According the automatic grading, there were 100 first-level fruit, 117 second-level fruit, and 87 third-level fruit. We then counted the number of wrinkle regions, to obtain the experimental result shown in Figure 7.

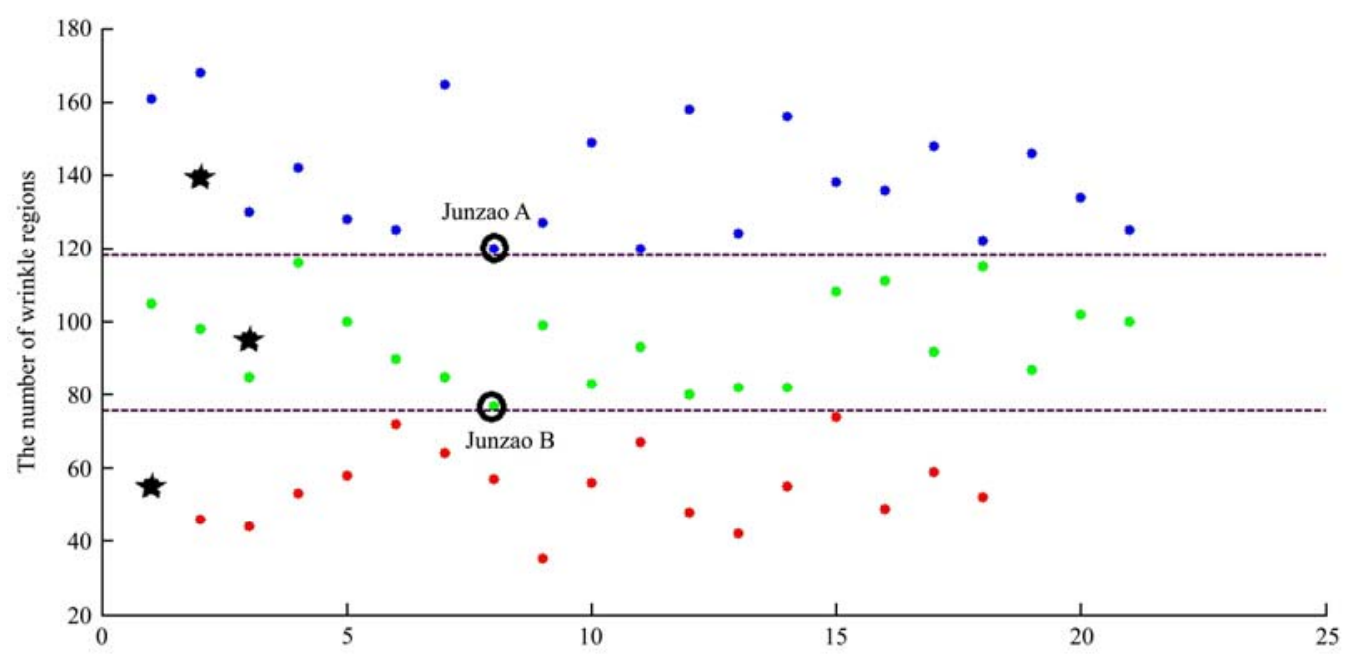

Figure 6 Choice of classification threshold from 60 samples

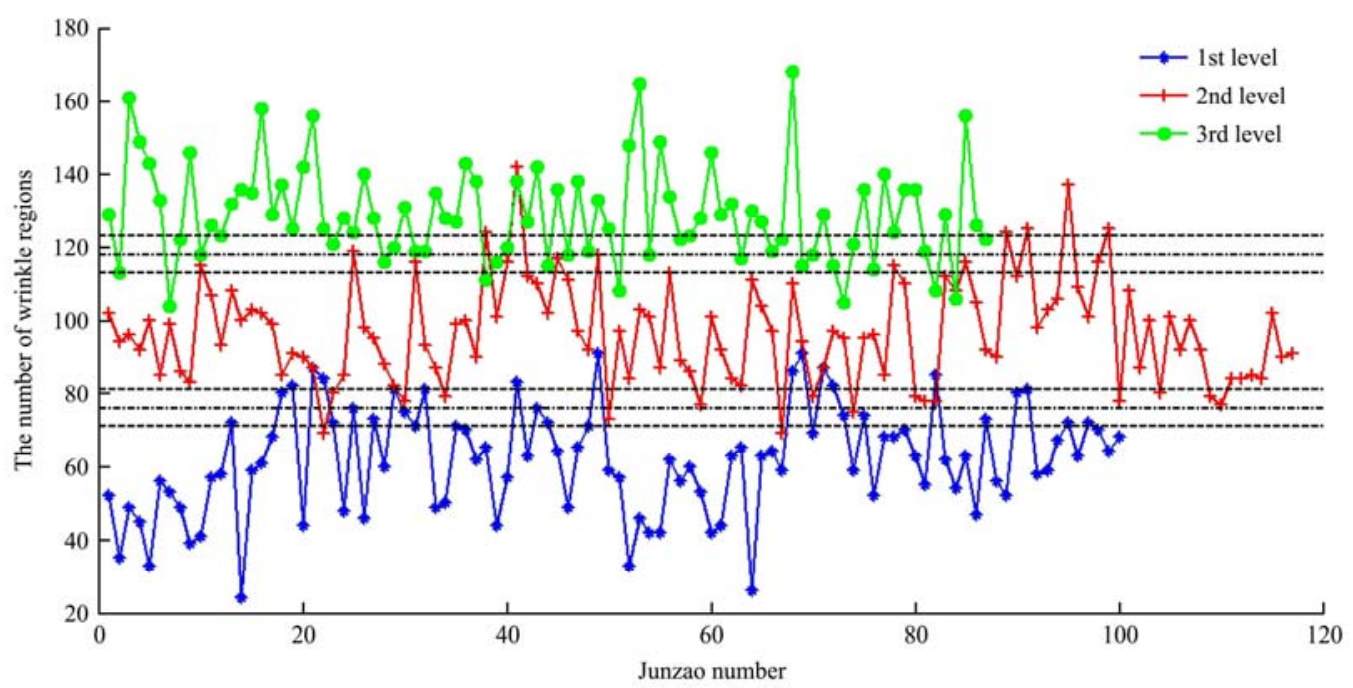

Figure 7 Experimental results of 304 samples

\subsection{Results analysis and feature description}

There was a one-to-one relationship between the regions that were extracted with the algorithm and the wrinkle regions on the surface of the jujube fruit. Therefore, the number of wrinkle regions segmented by the proposed algorithm could be used to describe the degree of wrinkles on a jujube fruit.

Figure 7 shows that most of the jujubes were graded correctly, but some of them were misclassified to the adjacent level because the number of wrinkle regions was close to the threshold. The reason why a small portion of jujube fruit misjudged was that the wrinkle degree 
could not be quantitatively distinguished and there were some errors in the automatic classification. Therefore, if $[-5,+5]$ is used as the allowable error range, the results are correct. Figure 5 shows that if the number of wrinkle regions are equal to or less than 81 , the jujube fruit in the image is classified as first-level grade. Moreover, if the number is within the range [71, 123], it is regarded as second-level grade. Once the wrinkles number 113 or more, the fruit is classified as third-level grade. The number of samples that were graded correctly and the accuracy of the classification are shown in Table 2, which shows that the jujube fruit grading accuracy rate for each of the three grades is $91.00 \%$, $93.16 \%$ and $93.10 \%$. Overall, for all 304 samples of jujube fruit, the accuracy rate of the classification is $92.43 \%$. In conclusion, the number of wrinkle regions on the surface of the jujube fruit can be used as a classification criterion to determine the degree of wrinkles.

Table 2 Results of sample grading

\begin{tabular}{ccccc}
\hline Grade & Sample size & Grading threshold & Exact quantity & Accuracy/\% \\
\hline 1 & 100 & $\mathrm{G}_{1}<76$ & 91 & 91.00 \\
2 & 117 & $76 \leq \mathrm{G}_{2} \leq 118$ & 109 & 93.16 \\
3 & 87 & $\mathrm{G}_{3}>118$ & 81 & 93.10 \\
Total & 304 & & 281 & 92.43 \\
\hline
\end{tabular}

\section{Conclusions}

In this study, to describe the degree of wrinkles on a jujube fruit, an automatic watershed segmentation-based grading method was used to extract the wrinkle features in a jujube fruit image taken in cyan light. Classification thresholds of 76 and 118 were selected by the automatic classification. Moreover, 304 samples of Xinjiang Hami jujube fruit were detected and their degree of wrinkles graded with a classification accuracy rate of $92.43 \%$. The results of the experiments showed that the number of jujube fruit wrinkle regions obtained using a marked watershed segmentation could be used as a classification criterion to determine the degree of wrinkles on the fruit.

\section{Acknowledgments}

The authors acknowledge that this research was financially supported by National Key Research Program
(2016YFD0701501) and Beijing Higher Education Young Elite Teacher Project (YETP0318).

\section{[References]}

[1] Liang H. On the actuality, existing problems and solutions to the industry of the Chinese jujube. Master's dissertation. Xi'an: Shaanxi Normal University, 2006; 47p. (in Chinese)

[2] Abbas M F, Al-Niami J H, Al-Sareh E A. The effect of ethephon on the ripening of fruits of jujube. Journal of Horticultural Science, 1994; 69(3): 465-466.

[3] Cyong J C, Hanabusa K. Cyclic adenosine monophosphate in fruits of Zizyphus jujuba. Phytochemistry, 1980; 19(12): $2747-2748$.

[4] Lee D J, Archibald J K, Chang Y C, Greco C R. Robust color space conversion and color distribution analysis techniques for date maturity evaluation. Journal of Food Engineering, 2008; 88(3): 364-372.

[5] Dahjye L, Robert S, James A, Steve M C. Development of a machine vision system for automatic date grading using digital reflective near-infrared imaging. Journal of Food Engineering, 2008; 86(3): 388-398.

[6] Ohali Y A. Computer vision based date fruit grading system: design and implementation. Journal of King Saud University-Computer and Information Sciences, 2011; 23(1): 29-36.

[7] Xue J L, Zhang S J, Zhang J J. Simultaneous detection of external and internal quality parameters of Huping jujube fruits using hyperspectral imaging technology. Spectroscopy and Spectral Analysis, 2015; 35(8): 2297-2302.

[8] Li J B, Deng X W, Kan Z, Tian X S, Xie F. The method of automatic dried red Jujube hierarchy based on machine vision. Agricultural Mechanization Research, 2014; 2: 55-59. (in Chinese)

[9] Zhao J W, Liu S P, Zou X B, Shi J Y, Yin X P. Recognition of defect Chinese dates by machine vision and support vector machine. Transactions of the CSAM, 2008; 39(3): 113-116. (in Chinese)

[10] Luo H P, Lu Q P. Application of near-infrared topology method in the quality analysis of jujube of southern Xinjiang. Spectroscopy and Spectral Analysis, 2012; 32(3): 655-659.

[11] Wu L G, He J G, Liu G S, Wang S L, He X G. Detection of common defects on jujube using Vis-NIR and NIR hyperspectral imaging. Postharvest Biology and Technology, 2016; 112: 134-142.

[12] Wang L L. Research of non-destructive grading technology and detection equipment for Hami big jujubes based on computer vision. Master's dissertation. Shihezi: Shihezi University, 2013; pp.31-34. (in Chinese) 
[13] Xia Y. Research about inspection technology on external feature of Xinjiang dry jujube based on machine vision technology. Master's dissertation. Zhenjiang: Jiangsu University, 2013; pp.46-52. (in Chinese)

[14] Zhan Y. Research of Southern Sinkiang Jujube color classification method based on machine vision. Master's dissertation. Alaer: Tarim University, 2015; pp.37-44. (in Chinese)

[15] Yuan S G, Wang M, Pan J, Hu F, Li D Y. A seamline optimization approach based on watershed segmentation for aerial image mosaicking. Acta Geodaetica Et Cartographica Sinica, 2015; 44(10): 1108-1116. (in Chinese)

[16] Jones G. Image segmentation using texture boundary detection. Pattern Recognition Letters, 1994; 15(6): 533-541.
[17] Shi C K. Research on digital LED lighting controller in machine vision. Master's dissertation. Guangzhou: South China University of Technology, 2010; pp.62-71. (in Chinese)

[18] Gonzalez R C, Woods R E. Digital image processing Second Edition. New Jersey: Prentice Hall Press, 2002; pp.617-626.

[19] Yu W B. Image processing based on MATLAB. Beijing: Tsinghua University Publishing House Press, 2008; pp. 182-183. (in Chinese)

[20] Soille P. Morphological image analysis principles and applications. New York: Springer-Verlag Press, 2008; pp. $150-152$.

[21] GB/T 5835-2009. Dried Chinese jujubes. (in Chinese) 